\title{
DEVELOPMENT OF A RECONFIGURABLE CONTROL FOR AN SP-100 SPACE REACTOR
}

\author{
MAN GYUN NA* and BELLE R. UPADHYAYA ${ }^{1}$ \\ Department of Nuclear Engineering, Chosun University \\ 375 Seosuk-dong, Dong-gu, Gwangju 501-759, Korea \\ 'Department of Nuclear Engineering, The University of Tennessee \\ Knoxville, Tennessee 37996-2300, USA \\ "Corresponding author. E-mail : magyna@chosun.ac.kr
}

Received May 22, 2006

Accepted for Publication July 11, 2006

In this paper, a reconfigurable controller consisting of a normal controller and a standby controller is designed to control the thermoelectric (TE) power in the SP-100 space reactor. The normal controller uses a model predictive control (MPC) method where the future TE power is predicted by using support vector regression. A genetic algorithm that can effectively accomplish multiple objectives is used to optimize the normal controller. The performance of the normal controller depends on the capability of predicting the future TE power. Therefore, if the prediction performance is degraded, the proportional-integral (PI) controller of the standby controller begins to work instead of the normal controller. Performance deterioration is detected by a sequential probability ratio test (SPRT). A lumped parameter simulation model of the SP-100 nuclear space reactor is used to verify the proposed reconfigurable controller. The results of numerical simulations to assess the performance of the proposed controller show that the TE generator power level controlled by the proposed reconfigurable controller could track the target power level effectively, satisfying all control constraints. Furthermore, the normal controller is automatically switched to the standby controller when the performance of the normal controller degrades.

KEYWORDS : Genetic Algorithm, Model Predictive Control, Reactor Power Control, Reconfigurable Control, Sequential Probability Ratio Test, SP-100 Space Reactor, Support Vector Machines

\section{INTRODUCTION}

The SP-100 space reactor [1] is a fast spectrum lithiumcooled reactor system designed for use as an orbital power supply, a lunar or Martian surface power station, or a power supply for nuclear electric propulsion. It is designed to be scalable from $10 \mathrm{~s} \mathrm{kWe}$ to $100 \mathrm{~s} \mathrm{kWe}$ with a generic design of $100 \mathrm{kWe}$. The energy conversion system is based on a direct thermoelectric (TE) conversion mechanism. The TE SP-100 system is made up of a nuclear reactor, a heat transport system with associated pumps, a thermoelectric device to convert heat to electricity, and a radiator configuration system to reject waste heat into space.

The control system is a key element of the reactor design in terms of meeting space mission requirements such as economics, reliability, safety, survivability, and life expectancy. In land-based nuclear power plants, human operators are available to perform intelligent control functions necessary for both normal operation and during accident situations. However, for a space mission where an uncertain environment, rare events, and communication delays should be anticipated, all control functions must be achieved through a sophisticated control system with a limited degree of human intervention from Earth. In order to meet mission requirements and support missions with extended periods of operation, the space reactor control system needs to have the capability of performance monitoring and optimization. For instance, in deep-space missions, the reactor system is expected to have continuous, remote, and unattended operation for up to fifteen years. During such an extended period, many thermal and electric components may experience a significant level of degradation. In order to reduce mission costs, the operation parameters should be adjusted to optimize operational performance according to current operating conditions and be implemented by a reconfigurable control mechanism. Many studies have been conducted to control the SP-100 space reactor [2-4]. Whereas previous studies have focused on normal control of the reactor, the present paper deals with reconfigurable control of the SP-100 space reactor.

The MPC method has received much attention as a powerful tool for control of industrial process systems [511]. This method has many advantages over conventional 
infinite horizon control techniques because it is possible to handle input and state (or output) constraints in a systematic manner during the design and implementation of the control. In particular, because of the model predictive control concept, which repeats the optimization calculation after measuring inputs and outputs at every time step, it provides a suitable control strategy for nonlinear time varying systems. Therefore, the reconfigurable controller employs the MPC method for a normal controller and also uses the conventional PI control method for a standby controller in case the performance of the normal controller is degraded. The model predictive control method has been applied to nuclear engineering problems [3-4,12].

In this paper, the normal controller uses support vector machines (SVMs), which have been successfully employed to solve nonlinear regression and time series modeling problems [13-15]. SVM regression is used to predict the future output, which is needed in optimizing an objective functional for the model predictive control. The objective functional for MPC is minimized by a genetic algorithm (GA), which is widely used in optimization problems.

The performance of the normal controller depends on the prediction capability of the future TE power. Therefore, if the prediction performance is degraded, the PI controller of the standby controller begins to work instead of the normal controller. Performance deterioration is detected by a SPRT. A lumped parameter simulation model of the SP100 space reactor is used to verify the proposed reconfigurable controller.

\section{CONTROL SYSTEM DESIGN}

The performance of the model predictive controller for the SP-100 space reactor has been verified to be excellent [3-4] but may degrade during significant transient conditions and time-varying dynamics. In the present paper, in order to address this potential weakness, the normal controller is switched to a standby controller whenever the normal controller experiences degradation. This is accomplished by continuously monitoring the performance of a model predictive controller, i.e., the normal controller. The normal controller is designed using model predictive control methodology and the standby controller is designed using the conventional PI control method, which has been widely used in industrial applications.

\subsection{A Normal Controller Using the MPC Methodology}

The model predictive control problem is formulated by solving on-line a finite horizon open-loop optimal control problem subject to system dynamics and constraints. Figure 1 shows the basic concept of the model predictive control [7]. Based on measurements obtained up to time $t$, the future dynamic behavior of the process outputs are predicted over a prediction horizon, assuming some trajectory of control

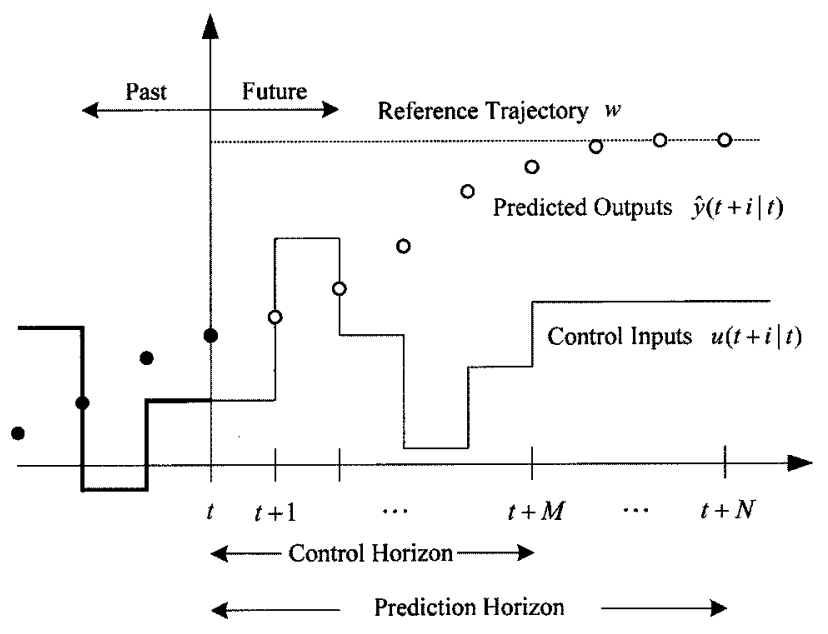

Fig. 1. Basic Concept of the Model Predictive Control Method

inputs over a fixed number of future time instants, known as the time horizon. Present and future control inputs are then determined such that an open-loop performance objective functional is minimized. Only the first control input among the optimized control input trajectories is implemented. At the next time step, the outputs are measured and the calculations of the prediction and optimization are repeated using updated measurements.

The purpose of taking new measurements at each time step is to compensate for unmeasured disturbances and model inaccuracies. In order to achieve a fast response and prevent the need for excessive control effort, the performance objective functional is represented by the following quadratic function:

$$
J=\frac{1}{2} \sum_{k=1}^{L}[\hat{y}(t+k \mid t)-z(t+k)]^{2}+\frac{1}{2} \sum_{k=1}^{M} \rho[\Delta u(t+k-1)]^{2}
$$

subject to constraints: $\Delta u(t+k-1)=0$ for $k>M ; u_{\min } \leq u(t)$ $\leq u_{\max } ;|\Delta u(t)| \leq \Delta u_{\max }$, where the parameter $\rho$ determines the trade-off between the TE power (system output) error and control drum angle (control input) change between neighboring time steps, and $z$ is a setpoint (desired TE power) or reference sequence for the output signal. The estimate $\hat{y}(t+k \mid t)$ is the optimum $k$-step-ahead prediction of the system output based on data up to time $t . \Delta u$ is the input change between neighboring time steps. The parameters $L$ and $M$ are the prediction horizon and the control horizon, respectively. The prediction horizon represents the limit of the instant at which it is desired for the output to follow the reference sequence.

In order to obtain optimal control inputs, the system 
outputs are first predicted by using support vector machines (SVMs) for a function approximation. SVMs were first applied for function classification problems. However, with the introduction of Vapnik's $\varepsilon$-insensitive loss function [16], they have been extended and are now widely used in solving nonlinear regression problems. The basic concept of the SVM regression is to nonlinearly map original data $\mathbf{x}$ into a higher dimensional feature space. Hence, given a set of data $\left\{\left(\mathbf{x}_{i}, y_{i}\right)\right\}_{i=1}^{N}$ where $\mathbf{x}_{i}$ is the input vector to SVMs, $y_{i}$ is the actual output value, and $N$ is the total number of data patterns, the SVM regression function is expressed as

$$
y=f(\mathbf{x})=\sum_{i=1}^{N} w_{i} \phi_{i}(\mathbf{x})=\mathbf{w}^{T} \boldsymbol{\varphi}(\mathbf{x})+b,
$$

where $\mathbf{w}=\left[\begin{array}{llll}w_{1} & w_{2} & \cdots & w_{N}\end{array}\right]^{T}$,

$$
\boldsymbol{\varphi}=\left[\begin{array}{llll}
\phi_{1} & \phi_{2} & \cdots & \phi_{N}
\end{array}\right]^{T} \text {. }
$$

$\phi_{i}(\mathbf{x})$ is called the feature that is nonlinearly mapped from the input space $\mathbf{x}$. The parameters $\mathbf{w}$ and $b$ are a support vector weight and a bias, respectively, and are calculated by minimizing the following regularized risk function:

$$
R(\mathbf{w})=\frac{1}{2} \mathbf{w}^{T} \mathbf{w}+\lambda \sum_{i=1}^{N} L_{\varepsilon}\left(\mathbf{x}_{i}, y_{i}, f\right)
$$

where

$$
L_{\varepsilon}(\mathbf{x}, y, f)=|y-f(\mathbf{x})|_{\varepsilon}= \begin{cases}0 & \text { for }|y-f(\mathbf{x})|<\varepsilon \\ |y-f(\mathbf{x})|-\varepsilon & \text { otherwise }\end{cases}
$$

$\lambda$ and $\varepsilon$ are user-specified parameters and $L_{\sigma}(\mathbf{x}, y, f)$ is called the $\varepsilon$-insensitive loss function [16]. The loss equals zero if the estimated value is within an error level $\varepsilon$ (refer to Figure 2). The regularized risk function can be rewritten in the following constrained form:

$$
R\left(\mathbf{w}, \xi, \xi^{*}\right)=\frac{1}{2} \mathbf{w}^{T} \mathbf{w}+\lambda \sum_{i=1}^{N}\left(\xi_{i}+\xi_{i}^{*}\right)
$$

subject to the constraints

$$
\left\{\begin{array}{l}
y_{i}-\mathbf{w}^{T} \boldsymbol{\varphi}(\mathbf{x})-b \leq \varepsilon+\xi_{i}, \quad i=1,2, \cdots, N \\
\mathbf{w}^{T} \boldsymbol{\varphi}(\mathbf{x})+b-y_{i} \leq \varepsilon+\xi_{i}^{*}, \quad i=1,2, \cdots, N \\
\xi_{i}, \xi_{i}^{*} \geq 0, \quad i=1,2, \cdots, N
\end{array}\right.
$$

The constant $\lambda$ determines the trade-off between the flatness of $f(\mathbf{x})$ and the amount up to which deviations larger than

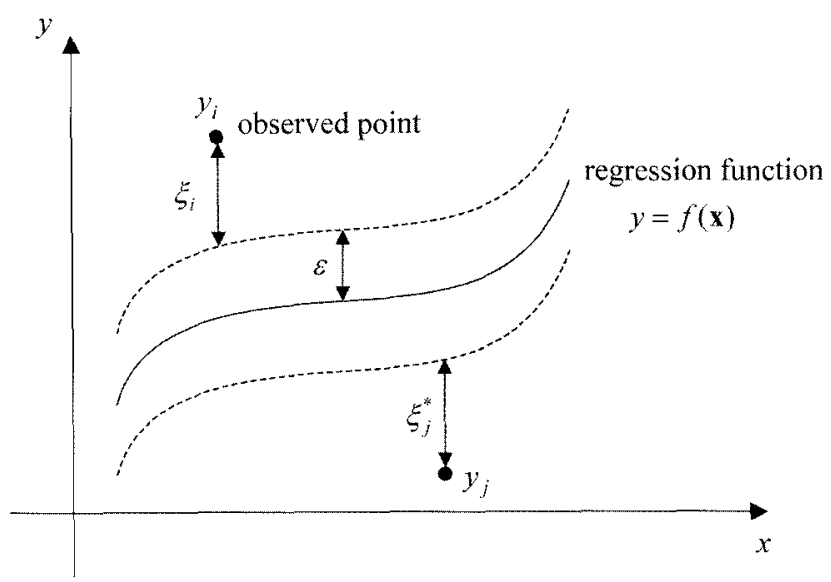

Fig. 2. Insensitive Band $( \pm \varepsilon)$ and Slack Variables $\xi$ and $\xi^{*}$ for the SVM Regression

$\boldsymbol{\varepsilon}$ are tolerated. $\xi=\left[\begin{array}{llll}\xi_{1} & \xi_{2} & \ldots & \xi_{N}\end{array}\right]^{T}$ and $\xi^{*}=\left[\begin{array}{llll}\xi_{1}^{*} & \xi_{2}^{*} & \ldots & \xi_{N}^{*}\end{array}\right]^{T}$ are slack variables representing the upper constraints and the lower constraints, respectively, on the outputs of the system.

The solution to the constrained optimization problem is given by the saddle point of the Lagrange functional [17]:

$\Phi\left(\mathbf{w}, b, \xi_{i}, \xi_{i}^{*}, \alpha_{i}, \alpha_{i}^{*}, \beta_{i}, \beta_{i}^{*}\right)$

$=\frac{1}{2} \mathbf{w}^{T} \mathbf{w}+\lambda \sum_{i=1}^{N}\left(\xi_{i}+\xi_{i}^{*}\right)-\sum_{i=1}^{N} \alpha_{i}\left[\mathbf{w}^{T} \boldsymbol{\varphi}\left(\mathbf{x}_{i}\right)+b-y_{i}+\varepsilon+\xi_{i}\right]$

$-\sum_{i=1}^{N} \alpha_{i}^{*}\left[y_{i}-\mathbf{w}^{T} \boldsymbol{\varphi}\left(\mathbf{x}_{i}\right)-b+\varepsilon+\xi_{i}^{*}\right]-\sum_{i=1}^{N}\left(\beta_{i} \xi_{i}+\beta_{i}^{*} \xi_{i}^{*}\right)$

The above equation is minimized with respect to the primal variables $\mathbf{w}, b, \boldsymbol{\xi}_{i}, \boldsymbol{\xi}_{i}^{*}$, and then maximized with respect to the nonnegative Lagrangian multipliers $\alpha_{i}, \alpha_{i}^{*}, \beta_{i}, \beta_{i}^{*}$. After going through a complex analysis procedure, the regression function of Equation (2) finally becomes

$$
\begin{aligned}
y=f(\mathbf{x}) & =\sum_{i=1}^{N}\left(\alpha_{i}-\alpha_{i}^{*}\right) \varphi^{T}\left(\mathbf{x}_{i}\right) \varphi(\mathbf{x})+b \\
& =\sum_{i=1}^{N}\left(\alpha_{i}-\alpha_{i}^{*}\right) K\left(\mathbf{x}, \mathbf{x}_{i}\right)+b
\end{aligned}
$$

where $K\left(\mathbf{x}, \mathbf{x}_{i}\right)=\varphi^{T}\left(\mathbf{x}_{i}\right) \varphi(\mathbf{x})$ is called the kernel function. A number of coefficients $\alpha_{i}-\alpha_{i}^{*}$ are nonzero values. The 
corresponding training data points have approximation error equal to or larger than $\varepsilon$ and are called support vectors.

From Equation (8), the current predicted output can be expressed generally as follows:

$$
\hat{y}(t / t)=f(\mathbf{x}(t))
$$

where $\mathbf{x}(t)=\left[y\left(t-n_{d}-1\right), \cdots, y\left(t-n_{d}-n_{y}\right), \mathrm{u}(t-1), \cdots\right.$, $\left.\mathrm{u}\left(t-n_{u}\right), \Delta u(t-1), \ldots, \Delta u\left(t-n_{\Delta u}\right)\right]$. The SVM-based future output prediction can then be calculated as

$$
\hat{y}(t+k / t)=d(t / t)+f(\mathbf{x}(t+k)), k=1, \cdots, L
$$

where the disturbance estimate $d(t / t)$ is assumed to be constant over the prediction horizon $L$ and is calculated as follows:

$$
d(t / t)=y(t)-\hat{y}(t / t)
$$

The disturbance estimate is used to correct the model forecast and to obtain offset-free control.

In this paper, we propose an SVM-based MPC methodology that is based on a dynamic nonlinear SVM model to design the normal controller for the SP-100 space reactor. The optimization problem, which needs to be solved online, is no longer a linear problem but a complicated nonlinear problem that requires a large amount of computational effort. That is, the proposed method formulates a dynamic nonlinear optimization problem where the objective function consists of two terms: (i) differences between the SVM model-predicted values and the desired output trajectory over a prediction horizon; and (ii) the control energy over a control horizon. Also, the objective function is subject to the constraints on the input variables and input steps. This calculation cannot be completed on time even by fast computing systems [18]. Due to the complexity of the SVM model, conventional optimization techniques cannot be easily applied.

Therefore, in this paper, the online optimization problem is solved using a genetic algorithm (GA). The GA is known to be effective in solving multiple objective functions. Compared to conventional optimization methods that move from one point to another, GAs start from many points, simultaneously climbing many peaks in parallel. Accordingly, GAs are less susceptible to getting stuck at local minima compared to conventional search methods [19-20]. In the GA, the term chromosome is a candidate solution that minimizes a cost function. As the generation proceeds, populations of chromosomes are iteratively altered by biological mechanisms inspired by natural evolution such as selection, crossover, and mutation. The GAs require a fitness function that assigns a score to each chromosome (candidate solution) in the current population, and maximizes the fitness function value. The fitness function evaluates the extent to which each candidate solution is suitable for specified objectives. The GA starts with an initial population of chromosomes, which represent possible solutions of the optimization problem. The fitness function is computed for each chromosome. New generations are produced by the genetic operators, such as selection, crossover, and mutation. The algorithm stops after the maximum allowed time has elapsed.

A chromosome is represented by $s_{g}$, whose elements consist of present and future control inputs and has the following structure $[18,21]$ :

$$
s_{g}=\left[u_{g}(t) u_{g}(t+1) \cdots u_{g}(t+M-1)\right], g=1, \cdots, G,
$$

where $t$ indicates the current time. Assuming we have chosen the number of chromosomes $G$, which will constitute the initial population, the crossover probability $p_{c}$, and the mutation probability $p_{m}$, the algorithm proceeds according to the following steps:

Step 1 (initial population generation): Set the number of iterations iter $=1$. Generate an initial population consisting of a total of $G$ chromosomes. The values are allocated randomly, but they should satisfy both the input and input move constraints of Equation (1).

Step 2 (fitness function evaluation): Evaluate the objective function of Eq. (1) for all the chosen chromosomes. Then invert the objective function values and find the total fitness of the population as follows :

$$
F=\sum_{g=1}^{G} \frac{1}{J_{g}(t)},
$$

where $J_{g}(t)$ is the objective function value for the $g$-th chromosome and the inversion of $J_{g}(t)$ is a fitness value of the $g$-th chromosome. Then, calculate the normalized fitness value of each chromosome, that is, the selection probability $p_{g}$, as follows :

$$
p_{g}=\frac{\left(1 / J_{g}(t)\right)}{F}, \quad g=1, \cdots, G
$$

Step 3 (selection operation): Calculate the cumulative probability $q_{g}$ for each chromosome using the following equation : 


$$
q_{g}=\sum_{j=1}^{g} p_{j}, \quad g=1, \cdots, G
$$

For $g=1, \cdots, G$, generate a random number $r$ between 0 and 1. Select the chromosome for which $q_{\mathrm{g}-1} \leq r \leq q_{\mathrm{g}}$. At this point of the algorithm a new population of chromosomes has been generated. The chromosomes with high fitness values have a greater chance of being selected.

Step 4 (crossover operation): For each chromosome $s_{g}$, generate a random number $r$ between 0 and 1 . If $r$ is lower than $p_{c}$, this particular chromosome will undergo the process of crossover; otherwise it will remain unchanged. Mate the selected chromosomes, and for each selected pair generate a random integer number $z$ between 0 and $M-1$. The crossing point is the position indicated by the random number. Two new chromosomes are produced by interchanging all the members of the parents following the crossing point. The crossover operation might produce infeasible offspring if the input values at the crossing point do not satisfy the input move constraints. Therefore, the crossover operation is conducted only if the input step constraints are satisfied.

Step 5 (mutation operation): For every member of each chromosome $s_{g}$, generate a random number $r$ between 0 and 1. If $r$ is lower than $p_{m}$, this particular member of the chromosome will undergo the process of mutation; otherwise it will remain unchanged. Each chromosome should satisfy both the input and input move constraints of Equation (1) after mutation. Therefore, the mutation operation is conducted only if the input move constraints are satisfied.

Step 6 (repeat or stop): If the maximum allowed time has not expired, set iter $=$ iter +1 and return to Step 2 . Otherwise, stop the algorithm and select the chromosome that produced the lowest value of the objective function throughout the entire procedure.

Although genetic algorithms usually require long computation time, the above-simplified GA makes it possible to calculate the optimal control in real time.

\subsection{A Standby Controller Using a PI Control Methodology}

The PI controller design is used extensively in industrial process control. The conventional PI method has advantages of easy implementation and well-proven technology. In this paper, a PI controller is designed to substitute for the normal controller in case of degraded performance of the normal controller. The discrete PI control logic is as follows:

$$
u(k)=u(k-1)+K_{p}(z(k)-y(k))+K_{I} T(z(k)-y(k))
$$

where $z(k)$ is a setpoint, $y(k)$ is a measured output, and $T$ is a sampling interval.

\section{CONTROLLER PERFORMANCE MONITORING}

The performance of the MPC controller depends heavily on the quality of the output prediction. Therefore, the output prediction error can be a good measure in monitoring the performance of the normal controller. At every new sample of the prediction error signal, a new mean and a new variance of the signal are required to assess the performance degradation of the normal controller. However, this procedure requires a large number of samples to calculate a meaningful mean and variance. Therefore, SPRT is used to detect the performance degradation based on the degree of degradation and the continuous behavior of the normal controller, without having to calculate a new mean and a new variance at each sampling instant. The SPRT is a statistical method developed by Wald [22-23].

The performance degradation of the normal controller should be detected as soon as possible with a very small probability of making a wrong decision. The SPRT uses the residuals (difference between the measured output and the predicted output, $y(k)-y(k))$. Normally, the residual sequence is randomly distributed, and the residuals are nearly uncorrelated and have a Gaussian distribution function $P_{i}\left(\varepsilon_{k}, m_{i}, \sigma_{i}\right)$ under hypothesis $i$, where $\varepsilon_{k}$ is the residual signal at time instant $k$. The performance degradation can be described in terms of the change in the mean $m$ or the change in the standard deviation $\sigma$. Therefore, the SPRT detects controller performance degradation by sensing alteration of the probability distribution. If a set of samples is collected with a density function describing each sample in the set, the overall likelihood ratio is given by

$$
\gamma_{n}=\frac{P_{1}\left(\varepsilon_{1} \mid H_{1}\right) \cdot P_{1}\left(\varepsilon_{2} \mid H_{1}\right) \cdot P_{1}\left(\varepsilon_{3} \mid H_{1}\right) \cdots P_{1}\left(\varepsilon_{n} \mid H_{1}\right)}{P_{0}\left(\varepsilon_{1} \mid H_{0}\right) \cdot P_{0}\left(\varepsilon_{2} \mid H_{0}\right) \cdot P_{0}\left(\varepsilon_{3} \mid H_{0}\right) \cdots P_{0}\left(\varepsilon_{n} \mid H_{0}\right)}
$$

where $H_{0}$ represents the hypothesis that the normal controller is working well and $H_{1}$ represents the hypothesis that its performance is degraded.

By taking the logarithm of the above equation and replacing the probability density functions in terms of residuals, means, and standard deviations (for a Gaussian density), the $\log$ likelihood ratio can be written in the following recurrent form:

$$
\lambda_{n}=\lambda_{n-1}+\ln \left(\frac{\sigma_{0}}{\sigma_{1}}\right)+\frac{\left(\varepsilon_{n}-m_{0}\right)^{2}}{2 \sigma_{0}^{2}}-\frac{\left(\varepsilon_{n}-m_{1}\right)^{2}}{2 \sigma_{1}^{2}}
$$

When the normal controller is working well, the log likeli- 
hood ratio will decrease and eventually reach a specified bound $A$, a value smaller than zero. When the ratio reaches this bound, the decision is made that the controller is working well, and the ratio is then reinitialized by setting it equal to zero. However, when the normal controller is working abnormally, the ratio will increase and eventually reach a specified bound $B$, a value larger than zero. When the ratio is equal to $B$, the decision is made that the controller is degraded. The decision boundaries $A$ and $B$ are chosen by a false decision probability $\alpha$ and a missed decision

probability $\beta ; A=\ln \left(\frac{\beta}{1-\alpha}\right)$ and $B=\ln \left(\frac{1-\beta}{\alpha}\right)[22]$.

\section{A DYNAMIC MODEL OF THE SP-100 SPACE REACTOR}

The SP-100 system is a fast spectrum lithium-cooled reactor system that can generate electric power of $100 \mathrm{~kW}$. The reactor system is made up of a reactor core, a primary heat transport loop, a thermoelectric generator, and a secondary heat transport loop to reject waste heat into space through radiators (refer to Figure 3 ). The reactor core is composed of small disks of highly enriched $(93 \%)$ uranium nitride fuel contained in sealed tubes. The heat generated in the reactor core is transported by liquid lithium pumped by sealed electromagnetic (EM) pumps. The interface between the primary heat transport system and the energy conversion system is a set of primary heat exchangers. The energy conversion system is based on a direct $\mathrm{TE}$ conversion mechanism. A temperature drop of about $500 \mathrm{~K}$ is maintained across the TE elements by the cooling effect of a second liquid lithium loop that transfers the waste heat from the converter to a heat-pipe radiator.

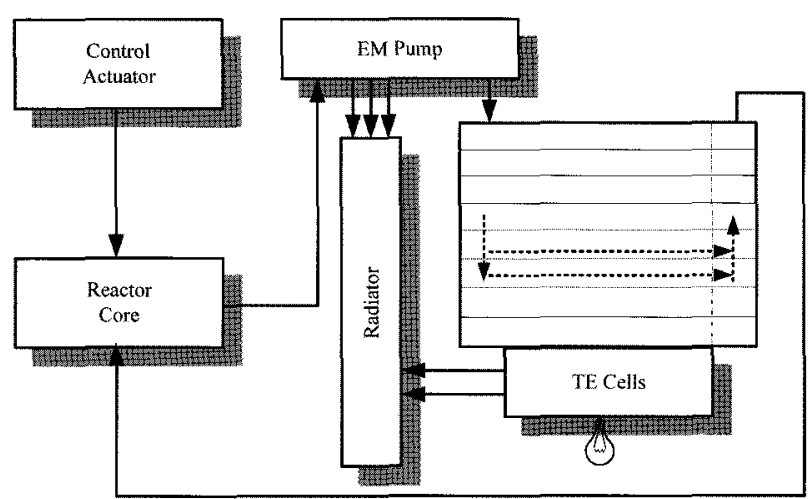

Fig. 3. Schematic of the Thermo-Electric SP-100 System [4]

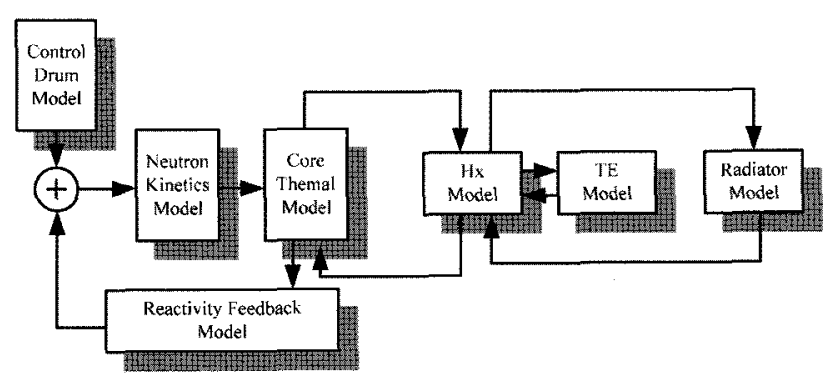

Fig. 4. Integrated Model of the SP-100 System [4]

A lumped parameter simulation model [24] was developed for the TE SP-100 system based on the early work of Seo [25]. The individual modules of the integrated model, as shown in Figure 4, include a model of the reactor control mechanism, a neutron kinetics model, a reactor core heat transfer model, and a heat exchanger model coupled with the TE conversion model. The integrated model involves a set of coupled nonlinear ordinary differential equations.

A point reactor kinetics model with six delayed neutron groups is used to describe the dependence of nuclear reactor power on the reactivity change. The core model takes into account three mechanisms of reactivity feedback: fuel Doppler feedback, reactor core expansion feedback, and reactor core coolant temperature feedback. The external reactivity control in the developed model uses the mechanism of the stepper motor control drum system [2]. The control drum shaft can be rotated from 0 to 180 degrees. The control voltage is transformed into a set of $27 \mathrm{~V}$ rectangular pulses with frequency varying from 0 to 1.33 $\mathrm{Hz}$. These pulses are then converted into discrete movements of the shaft connected to the control drums.

A simplified reactor core heat transfer model calculates the fuel temperature, the cladding temperature, and the average core coolant temperature. In the SP-100 reactor system, the reactor coolant enters the reactor vessel and flows up through the annular space between the reactor vessel and the core baffle until it reaches the upper plenum. At the upper plenum, the reactor coolant reverses and flows downward through the triangular fuel channels into the lower plenum. The total pressure drop in the reactor core consists of the pressure drop in the annular space, the pressure drop in the triangular fuel channel, the pressure drop in the upper plenum, and the pressure drop in the lower plenum. Because the available hydraulic data is limited, the simulation model has only explicitly dealt with the pressure loss in the annular space and in the triangular fuel channel.

The electric power is generated by 3 loops $\times 12$ primary heat exchangers $\times 30$ channels $\times 480$ TE cells. The hot shoes of the TE cells are mounted on the two surface plates 
of the 12 primary heat exchangers. The cold shoes of the TE cells are maintained at a lower temperature by cooling effects of the secondary lithium loop, which is connected to radiators through heat pipes. Because there is a temperature gradient between the hot shoes and the cold shoes of the TE cells, when heat is conducted from the hot shoe of a TE cell to its cold shoe, electric power is generated due to the Seebeck effect [26]. A single node for the radiator model is used to describe the heat removal by the radiators. It is assumed that the characteristic temperature of the coolant in the radiator is equal to the outlet temperature.

Table 1 presents a comparison between a current dynamic model [24] and SNPSAM [25] at the rated operation conditions. Most of the simulation results are in good agreement with the SNPSAM design parameters. Noticeable differences are shown for the TE hot side and cold side temperatures. The differences are attributed to the addition of a secondary lithium loop to the developed simulation model whereas TE cold shoes are directly connected to radiators in the SNPSAM design. Nevertheless, the calculated electric conversion efficiency is close to the value of SNPSAM. Therefore, the developed simulation model can be used to design a credible controller for the SP-100 space reactor system.

Table 1. Comparison Between SNPSAM and a Current Dynamic Model [4]

\begin{tabular}{c|c|c}
\hline Parameters & $\begin{array}{c}\text { SNPSAM } \\
\text { Design }[24]\end{array}$ & $\begin{array}{c}\text { Current } \\
\text { Model }\end{array}$ \\
\hline Nominal thermal power $(\mathrm{kW})$ & 2000.0 & 2000.3 \\
Electric power output $(\mathrm{kW})$ & 112.0 & 112.6 \\
Thermal efficiency $(\%)$ & 5.59 & 5.63 \\
Core inlet temperature $(\mathrm{K})$ & 1254.0 & 1254.0 \\
Core outlet temperature $(\mathrm{K})$ & 1284.0 & 1284.0 \\
Fuel temperature $(\mathrm{K})$ & 1376.0 & 1376.0 \\
Cladding temperature $(\mathrm{K})$ & 1288.0 & 1288.0 \\
TE hot side temperature $(\mathrm{K})$ & 1237.0 & 1228.5 \\
TE cold side temperature $(\mathrm{K})$ & 857.0 & 841.8 \\
\hline
\end{tabular}

\section{APPLICATION TO THE SP-100 SPACE REACTOR}

A schematic block diagram of the reconfigurable controller is presented in Figure 5. The controllers for the power level control of the SP-100 space reactor are subject to the following two constraints:

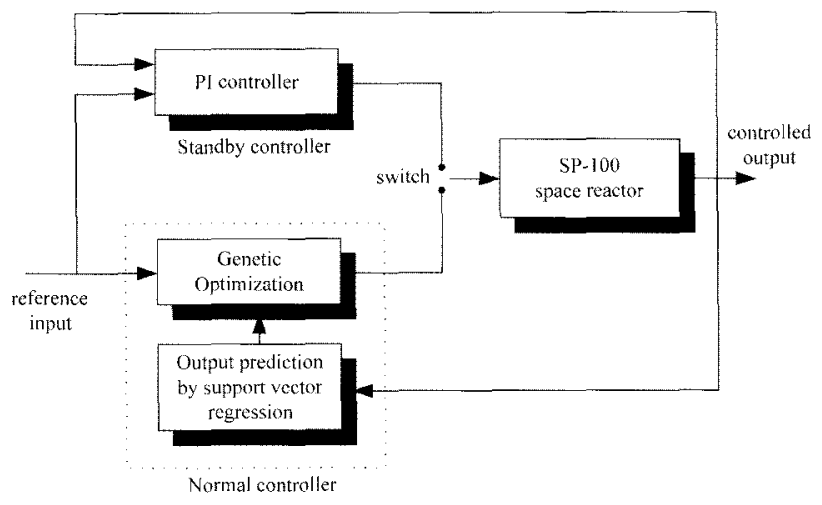

Fig. 5. Schematic Block Diagram of the Proposed Reconfigurable Controller

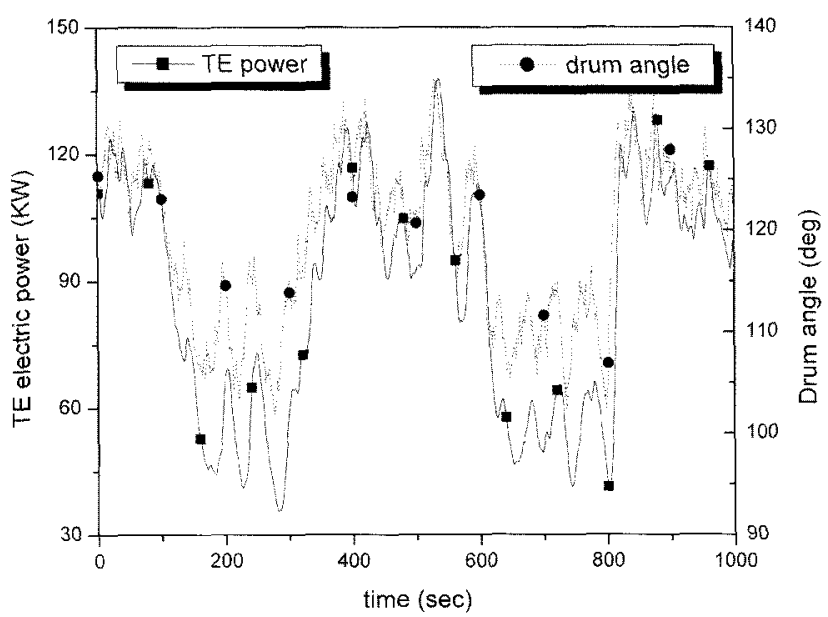

Fig. 6. Training Data Plot

$0^{\circ} \leq u(t) \leq 180^{\circ}$,

$|\Delta u(t)| \leq 1.4 T$

The sampling interval $T$ is 1 second. The external reactivity control uses the mechanism of the stepper motor control drum system [2]. The control drum angle of the stepper motor shaft can be rotated from 0 to 180 degrees. The maximum angular velocity of the drums is $1.4^{\circ} / \mathrm{sec}$.

The normal controller predicts the future TE generator power by using the SVM-based reactor model, which consists of the control drum angle and the past and current 


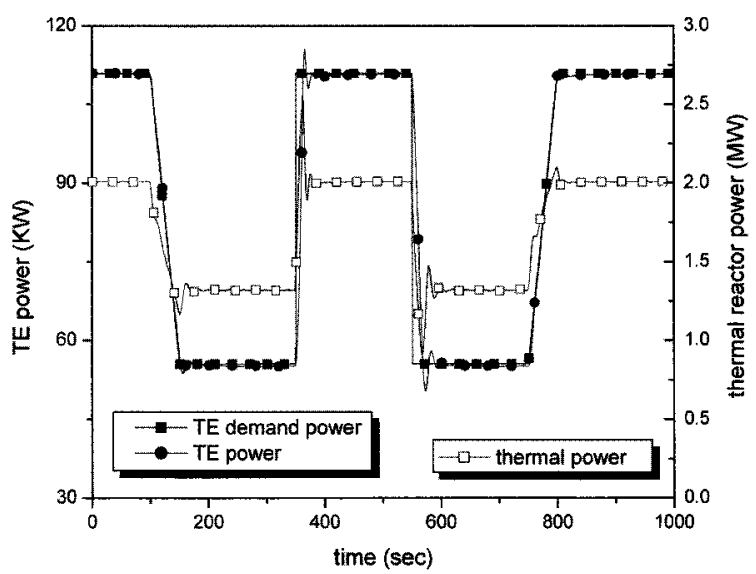

(a) TE power and thermal reactor power

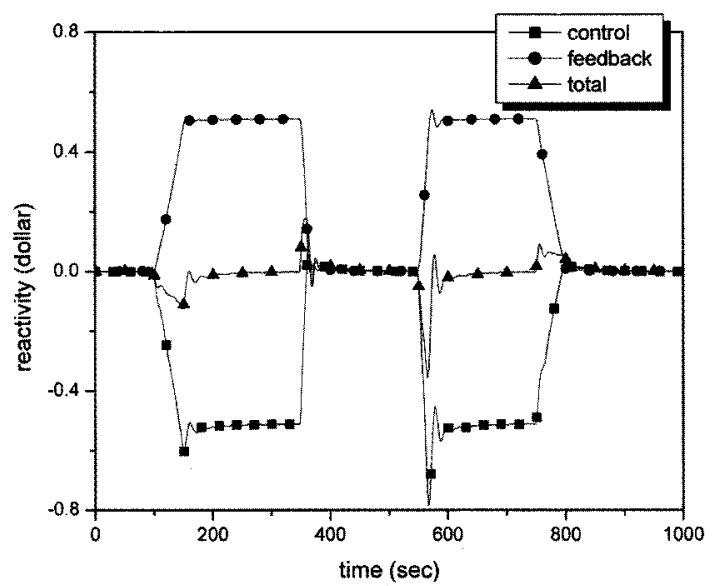

(c) Reactivity

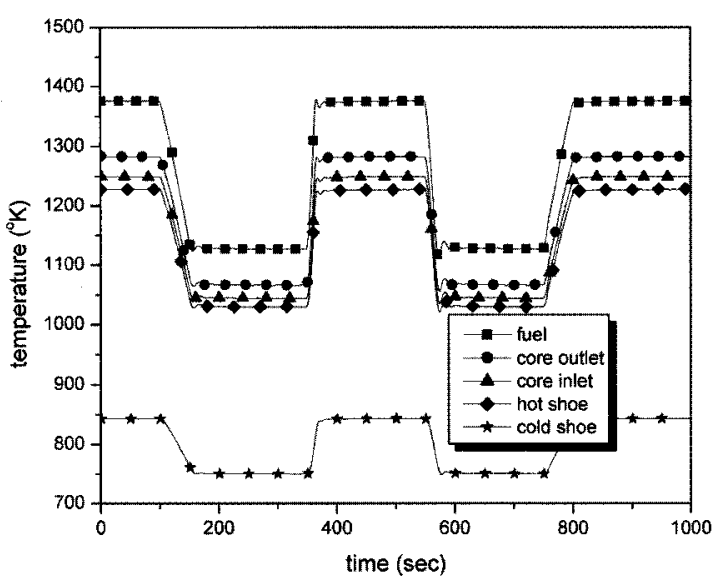

(e) Temperature

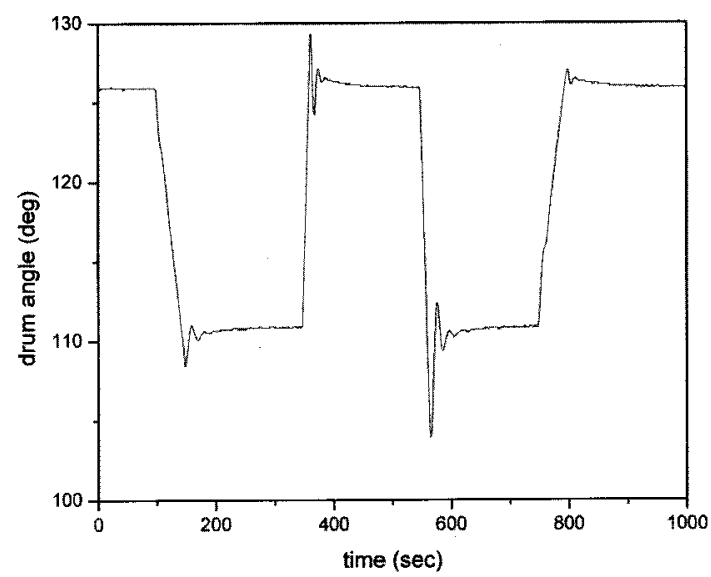

(b) Control drum angle

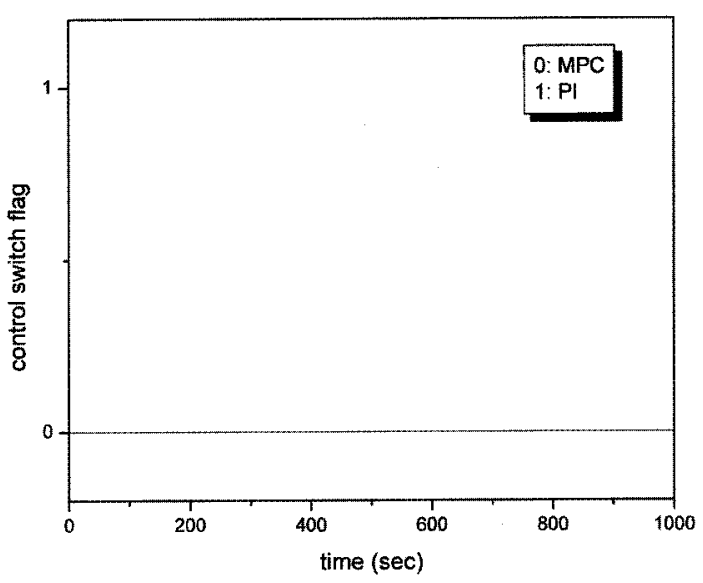

(d) Control switch flag

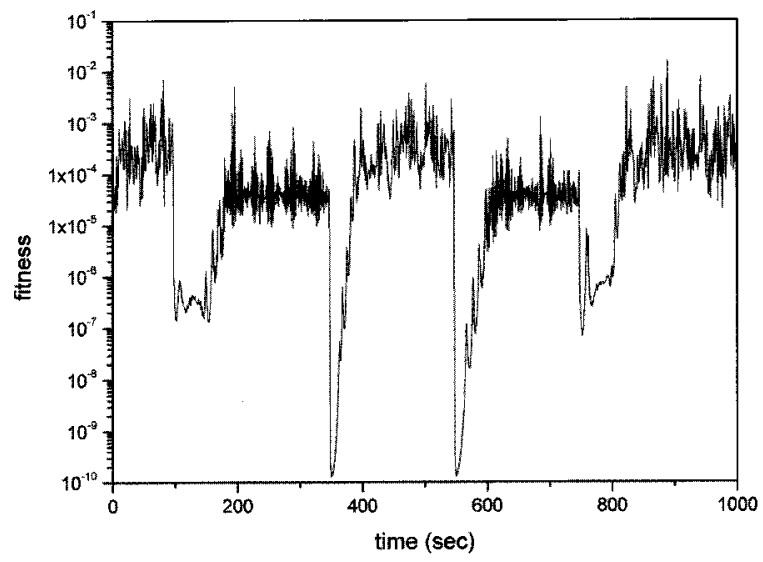

(f) Best fitness value of the genetic algorithm

Fig. 7. Performances of the Proposed Reconfigurable Controller for Power Transients 
TE generator power. The optimal control input is then calculated by minimizing the objective function of Equation (1) using the GA. The genetic algorithm usually converges at around the $30^{\text {th }}$ generation and stops unconditionally at the $50^{\text {th }}$ generation every time step. It takes about $0.7 \mathrm{sec}$ to evolute 50 generations for every time step (note that the sampling period is $1 \mathrm{sec}$ ). The following exponential radial basis function (ERBF) kernel is used in this paper:

$$
K\left(\mathbf{x}, \mathbf{x}_{i}\right)=\exp \left(-\left|\mathbf{x}-\mathbf{x}_{i}\right| / 2 \sigma^{2}\right) .
$$

The most relevant design parameters for the SVM regression model are the insensitivity zone $\varepsilon$, the regularization parameter $\lambda$, and the kernel parameter $\sigma$, which means kernel width. The SVM model parameters were chosen through many simulations. In general, an increase of the constant $\lambda$ penalizes larger errors, which leads to a decrease of approximation error. An increase in the insensitivity zone $\boldsymbol{\varepsilon}$ corresponds with a reduction in requirements for the accuracy of approximation and also decreases the number of support vectors, leading to data compression.

The regression function by SVMs is solved by using one-fifth of the data set shown in Fig. 6. Two hundred training data points are collected at every fifth interval (one per five data points) from the data of 1000 sampling points. Among these training data, 172 support vectors are determined.

Figure 7 shows the detailed simulation results of the proposed reconfigurable controller for power transients. The desired power is $100 \%$ initially and decreases to $50 \%$ by ramp from $100 \mathrm{sec}$ and recovers to $100 \%$ by step at 350 sec. Also, it decreases from $100 \%$ to $50 \%$ by step at $550 \mathrm{sec}$ and increases from $50 \%$ to $100 \%$ by ramp from $750 \mathrm{sec}$. It is shown that the TE generator power follows its desired value very well. It was found that the proposed reconfigurable controller meets several constraints very well and yields fast and stable responses. Figure $7(\mathrm{e})$ shows that the normal controller is not switched to the standby controller during the entire simulation time since the normal controller is not observed to be degraded. Figure 7(f) shows the trend of the best fitness function value, which is affected by the magnitudes of the estimated output error and the control input move. The fitness value decreases abruptly at the moment that the desired TE power changes, because large output error takes place.

Figure 8 shows the simulation results of the MPC controller and the PI controller for power transients (the same power transients were assumed for these simulations). As known from Figure 7(e), since only the normal controller (MPC controller) of the reconfigurable controller works well during all the simulation time, the response of the MPC controller is the same as that of the reconfigurable controller. As shown in Fig. 8(b), the response of the PI

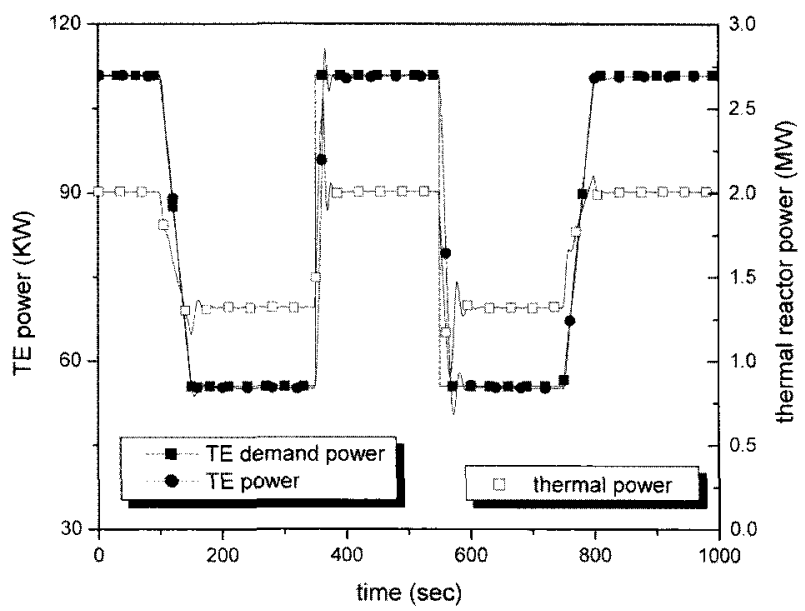

(a) MPC controller

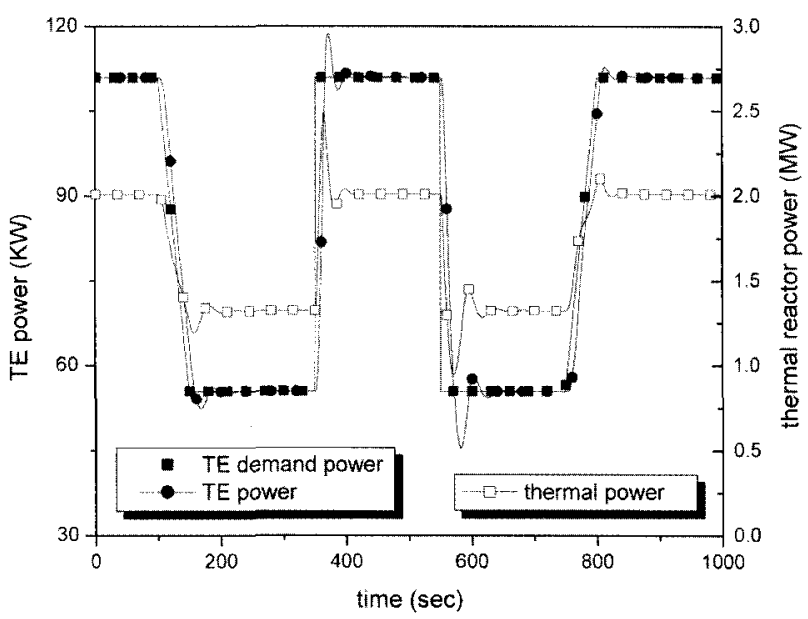

(b) PI controller

Fig. 8. Performances of Other Controllers for Power Transients

controller is delayed and the overshoot and undershoot of the PI controller response are larger than those of the MPC controller. The proportional gain $K_{p}$ is $4.0 \times 10^{-5}$ and the integral gain $K_{I}$ is $2.0 \times 10^{-6}$. These gains were chosen through many simulations.

Figure 9 shows the performance of the proposed reconfigurable controller in the case where a system dynamics parameter changes along with power transients. As shown in Fig. 9(c), the fuel temperature coefficient decreases by ramp from $400 \mathrm{sec}$ and increases by step at $700 \mathrm{sec}$. While the parameter deviates considerably from its normal value, the output prediction quality decreases. This in turn induces the normal controller to be switched to the standby (PI) 


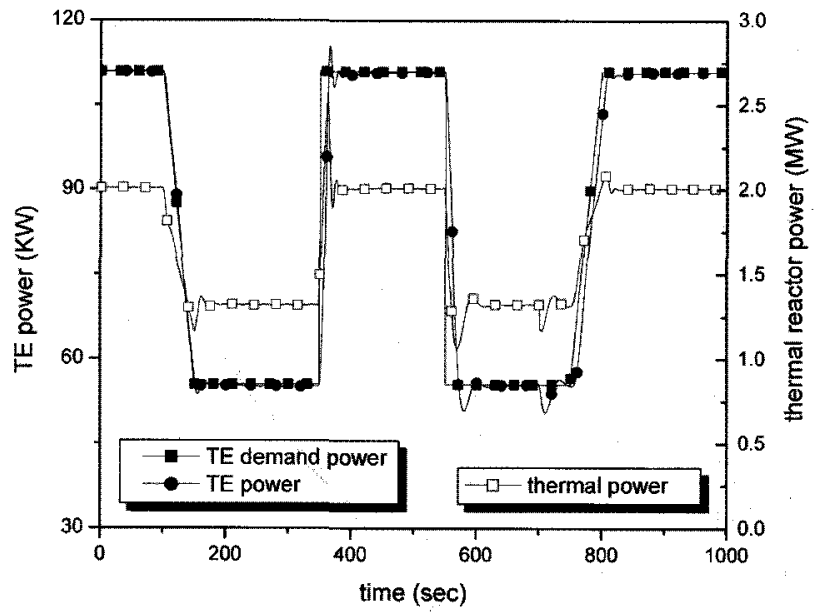

(a) TE power and thermal reactor power

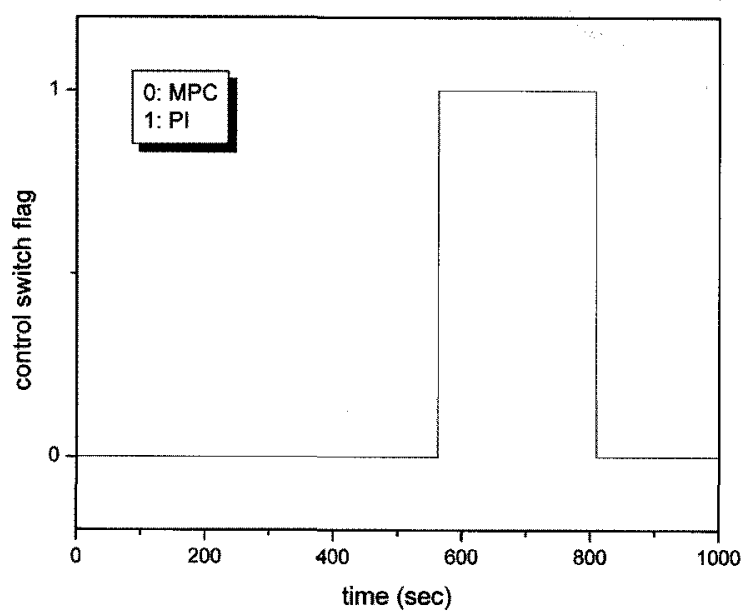

(b) Control switch flag

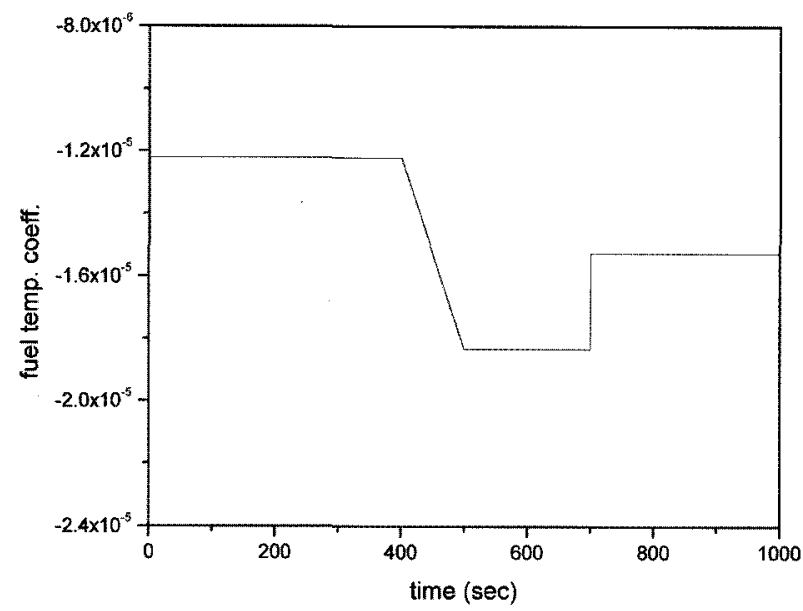

(c) Fuel temperature coefficient

Fig. 9. Performances of the Proposed Reconfigurable Controllers for Power Transients Accompanied by a Parameter Change

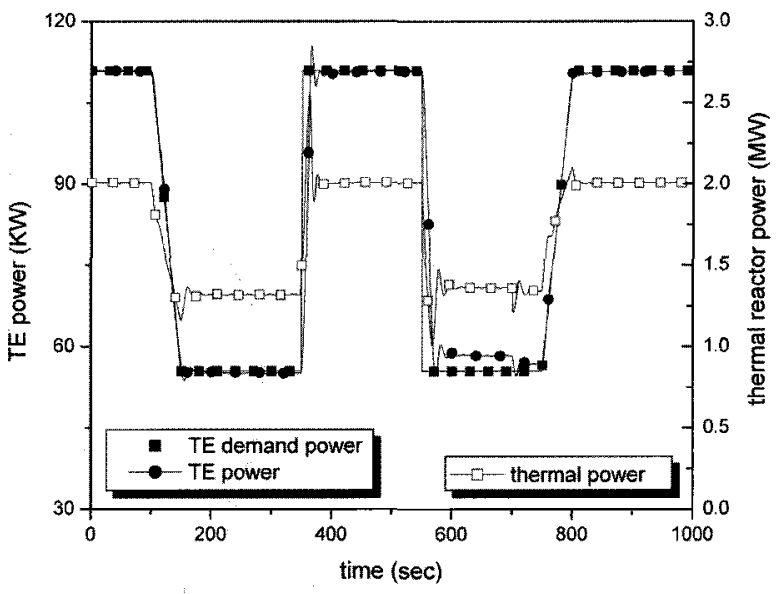

(a) MPC controller

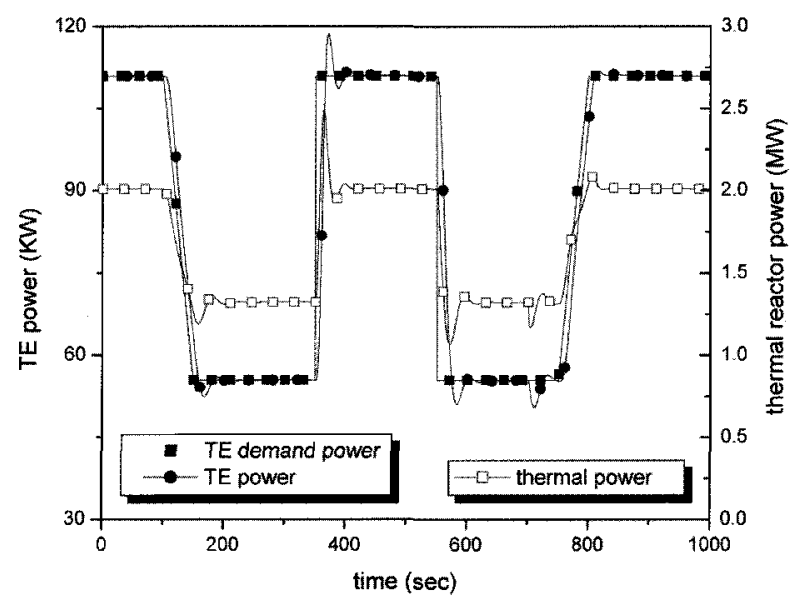

(b) PI controller

Fig. 10. Performances of Other Controllers for Power Transients Accompanied by a Parameter Change

controller. The undershoot response at $700 \mathrm{sec}$ is induced by the abrupt parameter change. The reconfigurable controller shows good performance in spite of a parameter change and power transients.

Figure 10 shows the simulation results of the MPC controller and the PI controller for power transients accompanied by a parameter change. The parameter change of this simulation is assumed to be the same as that of Figure 9. The change of the fuel temperature coefficient lowers the output prediction performance of the MPC controller, which degrades the MPC controller between $550 \mathrm{sec}$ and $750 \mathrm{sec}$. During this degraded period, the measured TE 
power does not follow its desired value. Except for at around the time $(700 \mathrm{sec})$ of the abrupt parameter change the PI controller performance is not significantly different from the simulation result of Figures $8(\mathrm{~b})$.

From simulations of setpoint changes such as a step increase and decrease and a ramp increase and decrease, and from simulations of parameter changes, it was found that the controlled output follows the setpoint change well and the controlled output is predicted well through a nonlinear identification model identified by the SVMs.

The proposed reconfigurable controller outperforms both the MPC controller and the PI controller in transient situations of step and ramp changes of the reference input as well as in transient situations accompanying a parameter change. The proposed control algorithm is coded with MATLAB language [27]. However, MATLAB calculation speed is very slow for computations that include the loop calculation. In spite of the slow loop calculation speed, it takes about $0.7 \mathrm{sec}$ to simulate $1 \mathrm{sec}$ by a $1.8 \mathrm{GHz}$ PC. Therefore, it can be implemented in real time.

\section{CONCLUDING REMARKS}

In this paper, a reconfigurable controller consisting of a normal controller and a standby controller was designed to control the TE power in the SP-100 space reactor. The normal controller uses a model predictive control method where the future TE power is predicted using SVM regression. A genetic algorithm was used to optimize the normal controller. In cases where the normal controller is degraded, the standby controller begins to work instead of the normal controller. Performance deterioration is detected by a SPRT. The TE generator power level controlled by the proposed reconfigurable controller could track the target power level effectively, satisfying all control constraints. Furthermore, the normal controller is automatically switched to the standby controller when the normal controller performance shows degradation.

\section{ACKNOWLEDGMENTS}

This study was supported in part by research funds from Chosun University, 2005.

\section{REFERENCES}

[1] S. F. Demuth, "SP-100 Space Reactor Design," Progress in Nuclear Energy, Vol. 42, No. 3, pp. 323-359, 2003.

[2] Y. B. Shtessel, "Sliding Mode Control of the Space Nuclear Reactor System," IEEE Trans. Aerospace and Electronic Systems, Vol. 34, No. 2, pp. 579-589, 1998.

[3 ] M. G. Na and B. R. Upadhyaya, "Model Predictive Control of an SP-100 Space Reactor Using Support Vector Regression and Genetic Optimization," IEEE Trans. Nucl. Sci., Vol. 53, No. 4, pp. 2318-2327, Aug. 2006.

[4] M. G. Na, B. R. Upadhyaya, X. Xu and I. J. Hwang, "Design of a Model Predictive Power Controller for an SP-100 Space Reactor," Nucl. Sci. Eng., Vol. 154, No.3, pp. 353-366, Nov. 2006.
[5] W. H. Kwon and A. E. Pearson, "A Modified Quadratic Cost Problem and Feedback Stabilization of a Linear System," IEEE Trans. Automatic Control, Vol. 22, No. 5, pp. 838842, 1977.

[6] J. Richalet, A. Rault, J. L. Testud and J. Papon, "Model Predictive Heuristic Control: Applications to Industrial Processes," Automatica, Vol. 14, pp. 413-428, 1978.

[ 7 ] C. E. Garcia, D. M. Prett and M. Morari, "Model Predictive Control: Theory and Practice - A Survey," Automatica, Vol. 25, No. 3, pp. 335-348, 1989

[ 8 ] D. W. Clarke and R. Scattolini, "Constrained RecedingHorizon Predictive Control," IEE Proceedings-D, Vol. 138 , No. 4, pp. 347-354, 1991.

[9] M. V. Kothare, V. Balakrishnan and M. Morari, "Robust Constrained Model Predictive Control Using Linear Matrix Inequality," Automatica, Vol. 32, No. 10, pp. 136l-1379, 1996.

[10] J. W. Lee, W. H. Kwon and J. H. Lee, "Receding Horizon $H^{\infty}$ Tracking Control for Time-Varying Discrete Linear Systems," Intl. J. Control, Vol. 68, No. 2, pp. 385-399, 1997.

[11] J. W. Lee, W. H. Kwon and J. Choi, "On Stability of Constrained Receding Horizon Control with Finite Terminal Weighting Matrix," Automatica, Vol. 34, No. 12, pp. 1607 $1613,1998$.

[12] M. G. Na, S. H. Shin and W. C. Kim, "A model predictive controller for nuclear reactor power," J. Korean Nucl. Soc., Vol. 35, No. 5, pp. 399-411, Oct. 2003.

[13] P.-F. Pai and W.-C. Hong, "Support Vector Machines with Simulated Annealing Algorithms in Electricity Load Forecasting," Energy Conversion and Management, Vol. 46, pp 2669-2688, 2005.

[14] W. Yan, H. Shao and X. Wang, "Soft Sensing Modeling Based on Support Vector Machine and Bayesian Model Selection," Computers and Chemical Engineering, Vol. 28, pp. 1489-1498, 2004.

[15] A. Kulkarni, V. K. Jayaraman and B. D. Kulkarni, "Control of Chaotic Dynamical Systems Using Support Vector Machines," Physics Letters A, Vol. 317, pp. 429-435, 2003.

[16] V. Vapnik, The Nature of Statistical Learning Theory, Springer, New York, 1995.

[17] H.W. Kuhn and A.W. Tucker, "Nonlinear programming," Proc. 2nd Berkeley Symp. Math. Stat. Probab., Univ. California Press, pp. 481-492, 1951.

[18] H. Sarimveis and G. Bafas, "Fuzzy Model Predictive Control of Non-Linear Processes Using Genetic Algorithms," Fuzzy Sets Systems, Vol. 139, pp. 59-80, 2003.

[19] D. E. Goldberg, Genetic Algorithms in Search, Optimization, and Machine Learning, Addison Wesley, Reading, MA, 1989.

[20] M. Mitchell, An Introduction to Genetic Algorithms, MIT Press, Cambridge, MA, 1996.

[21] M. G. Na and I. J. Hwang, "Design of a PWR Power Controller Using Model Predictive Control Optimized by a Genetic Algorithm," Nucl. Eng. Tech., Vol. 38, No. 1, pp. 81-92, Feb. 2006.

[22] A. Wald, Sequential Analysis, John Wiley \& Sons, New York, 1947.

[23] B. R. Upadhyaya, F. P. Wolvaardt and O. Glockler, "An Integrated Approach for Signal Validation in Dynamic Systems," Progress in Nuclear Energy, Pergamon Press, Vol. 21, pp. 605-611, 1988. 
[24] B. R. Upadhyaya and K. Zhao, "Dynamic Modeling and Control of Space Nuclear Power Systems," Proc. Space Nuclear Conference 2005, San Diego, California, June 5-9, 2005.

[25] J. T. Seo, "SNPSAM-Space Nuclear Power System Analysis Model," M.S. thesis, University of New Mexico, 1986.
[26] B.R. Upadhyaya, K. Zhao and X. Xu, Autonomous Control of Space Reactor Systems, Annual Report prepared for the U.S. Department of Energy NEER Program, Report No. DE-FG07-04ID14589/UTNE-02 (2005)

[27] MathWorks, MATLAB 5.3 (Release 11). Natick, MA: The MathWorks, 1999. 